Z Rheumatol 2010 - 69:9-10

DOI 10.1007/s00393-009-0597-1

Online publiziert: 16. Dezember 2009

(c) Springer-Verlag 2009

\author{
R.E. Schmidt ${ }^{1} \cdot$ T. Witte $^{1} \cdot$ T. Dörner ${ }^{2}$ \\ ${ }^{1}$ Klinik für Immunologie und Rheumatologie, Medizinische Hochschule Hannover \\ ${ }^{2}$ Medizinische Klinik mit Schwerpunkt Rheumatologie und Klinische \\ Immunologie, Charité-Universitätsmedizin Berlin, Campus Mitte \\ und Deutsches Rheumaforschungszentrum, Berlin
}

\title{
Das Sjögren-Syndrom
}

Behandlungsmöglichkeiten zur Verfügung stehen, können extraglanduläre Manifestationen durch den Einsatz von Kortikosteroiden, NSAR und/oder Immunsuppressiva z. T. sehr erfolgreich behandelt werden. In Pilotstudien wird derzeit als neuerer Therapieansatz die B-ZellDepletion geprüft, und notwendig sind sicher weitere Studien zur Entwicklung einer effektiven Behandlung des SjögrenSyndroms.

Eugen Feist und Arne Hansen stellen in ihrem Beitrag die Parameter und Messinstrumente vor, anhand derer der Behandlungserfolg des Morbus Sjögren beurteilt werden kann. Diese OutcomeParameter erlauben nicht nur die Beurteilung der Krankheitsaktivität und des Krankheitsverlaufes, sondern definieren auch Risikogruppen, die einer besonderen Überwachung bedürfen. Der Beitrag gibt einen kritischen Überblick über die verfügbaren bzw. in der Entwicklung befindlichen Outcome-Parameter für SjögrenPatienten, die ihrerseits von Relevanz für die valide Prüfung neuer Medikamente sind.

\section{- Der fachübergreifenden Zusammenarbeit kommt gerade beim Sjögren-Syndrom eine große Bedeutung zu.}

Daher ist der Beitrag von Christina Jakobi und Klaus Cursiefen aus Erlangen besonders zu begrüßen. In ihm stellen die Autoren die ophthalmologischen Komplikationen, die Möglichkeiten ihrer Diagnostik und Therapie zusammen. Unzureichende Therapiemaßnahmen können beim Sjögren-Syndrom zur Erblindung führen. In der Regel stehen die Störungen des präkornealen Tränenfilms im Vorder- grund. Durch die frühe und enge Kooperation zwischen Augenarzt und Rheumatologen können visusbedrohende Langzeitkomplikationen bei dieser Erkrankung leicht vermieden werden.

Wenn auch moderne epidemiologische Untersuchungen nach Gisela Westhoffs und Angela Zinks Mitteilungen nach den Amerikanisch-Europäischen-ConsensusGroup- (AECG-) Klassifikationskriterien nur eine Prävalenz von rund o,2\% für das primäre Sjögren-Syndrom finden, so ist doch in Deutschland zusammen mit den sekundären Sjögren-Syndrom-Patienten mindestens mit einer Prävalenz von $0,4 \%$ in der erwachsenen Bevölkerung mit immunpathologischer Sicca-Symptomatik auszugehen. Die Verteilung von 1:9 bezüglich Männer zu Frauen liegt nach neueren Daten eher bei 1:20. Dabei haben Frauen häufiger immunologische, histopathologische und sialographische Befunde und Organbeteiligungen.

Auch der Erkrankungsbeginn liegt nach den jüngeren Untersuchungen mit 45 Jahren 10 Jahre niedriger als früher festgestellt. Die im höheren Alter erkrankten Patienten sind häufiger Rheumafaktor- und Ro-/SS-A-positiv. Diese Veränderungen liegen sicher daran, dass die neue Diagnostik nach den modifizierten AECG-Kriterien einen großen Anteil von Patienten mit teils anderen immunologischen Markern und schwerwiegender Sicca-Symptomatik sowie Organbeteiligung von der Diagnose ausschließt.

Aber selbst nach diesen Daten werden höchstens 10\% der Patienten mit SjögrenErkrankung in Deutschland von Rheumatologen behandelt. Hier ist also auf jeden Fall eine sehr viel größere Aufmerksamkeit für die Diagnostik dieses Syndroms zu empfehlen. 
Torsten Witte aus Hannover geht auf die genetischen Risikofaktoren für das Sjögren-Syndrom wie STAT4, ILT-6 und den Haplotyp HLA-B8/DR3 ein. Zudem diskutiert er die für die Pathophysiologie wichtigen chronischen Virusinfektionen, die nach wie vor als bisher nicht erkannte Prognosefaktoren des Sjögren-Syndroms kandidieren. Zytotoxische T-Zellen zerstören die Speichel- und Tränendrüsen der Patienten, führen zu pathogenen $\mathrm{Zy}$ tokinen und Autoantikörpern mit nachfolgender Mund- und Augentrockenheit. Er stellt auch besonders die extraglandulären Manifestationen, die häufig schwierig diagnostiziert werden, wie Vaskulitis, Polyneuropathie oder Arthritis heraus und erläutert die diagnostischen Möglichkeiten beim Sjögren-Syndrom.

\section{( Höchstens 10\% der Patienten mit Sjögren-Erkrankung werden von einem Rheumatologen behandelt}

Wir hoffen, mit den Beiträgen dieses Heftes einen aktuellen Abriss des medizinischen Wissens zum Sjögren-Syndrom zu bieten, im Spannungsfeld der sehr interessanten und interdisziplinären Erkrankung mit der Darstellung des bisher Erreichten und den nach wie vor existierenden Erfordernissen.
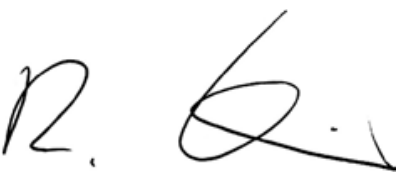

Reinhold E. Schmidt

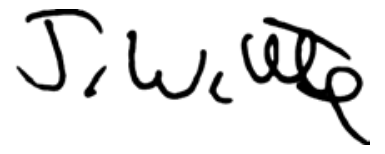

Torsten Witte

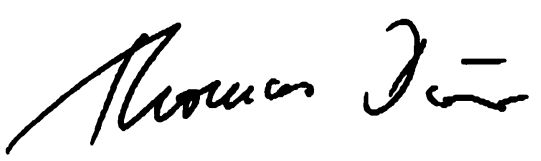

Thomas Dörner

\section{Korrespondenzadresse}

Prof. Dr. R.E. Schmidt

Klinik für Immunologie und Rheumatologie,

Medizinische Hochschule Hannover

Carl-Neuberg-Str. 1, 30625 Hannover

immunologie@mh-hannover.de

\section{Exklusiv für Abonnenten und Gesellschaftsmitglieder :}

\section{Nutzen Sie das Online-Archiv der Zeitschrift für Rheumatologie Ihre Vorteile: \\ - Komfortable und schnelle Recherche nach Themen, Autoren, Suchbegriffen \\ - Ob unterwegs oder am eigenen PC: Zugriff überall und jederzeit \\ - Online First: Lesen Sie die aktuellsten Beiträge schon vor Erscheinen des gedruckten Heftes online}

\section{Registrieren Sie sich jetzt unter \\ www.ZeitschriftfuerRheumatologie.de}

Mitglieder der Deutschen Gesellschaft für Rheumatologie haben direkten Zugriff auf die Volltexte über den Mitgliederbereich der Homepage der DGRh (www.dgrh.de)

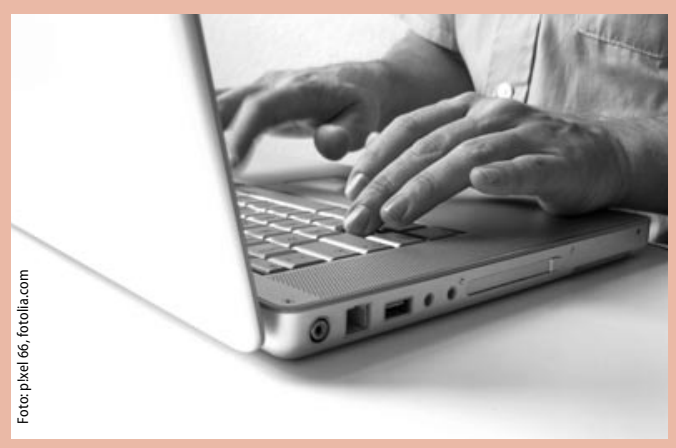

